\title{
Resonant Energy Transfer, with Creation of Hyper-Excited Atoms, and Molecular Auto-Ionization in a Cold Rydberg Gas
}

\author{
Marwan Rasamny, Alan Martinez, Lianxin Xin, Jun Ren, Essaid Zerrad \\ Division of Physics, Engineering, Mathematics, and Computer Science, Delaware State University, Dover, DE, USA \\ Email: ezerrad@desu.edu
}

How to cite this paper: Rasamny, M., Martinez, A., Xin, L.X., Ren, J. and Zerrad, E. (2021) Resonant Energy Transfer, with Creation of Hyper-Excited Atoms, and Molecular Auto-Ionization in a Cold Rydberg Gas. Journal of Modern Physics, 12, 1210-1218.

https://doi.org/10.4236/jmp.2021.129074

Received: May 23, 2021

Accepted: July 6, 2021

Published: July 9, 2021

Copyright $\odot 2021$ by author(s) and Scientific Research Publishing Inc. This work is licensed under the Creative Commons Attribution International License (CC BY 4.0).

http://creativecommons.org/licenses/by/4.0/

(c) (i) Open Access

\begin{abstract}
A cold Rydberg gas, with its atoms prepared initially all in the excited state $\left|n_{0}\right\rangle$, with $n_{0} \gg 1$, contains an excessive amount of energy, and presumably is to relax by the Penning-type molecular auto-ionization (MAI), in which a portion of excess energy of one atom is given to another near-by atom and ionizing it. Its complementary process, the resonant energy transfer (RET), is discussed, in which the excess energy of one atom is used on another to form a hyper-excited atomic state $\left|n_{a}\right\rangle$ with $n_{a} \gg n_{0}$. This process is always present, provided certain resonance energy conditions are satisfied. In this report, the $n_{0}$ and density dependences of the RET rates are studied in detail, employing a simple model: 1) at low densities, the RET is mediated by the dipole-dipole coupling $V_{d d}$ and its rates are generally much smaller than that of MAI, especially for small $n_{0}$. But 2) as the density increases, our model shows that the rates become of comparable magnitude or even larger than the MAI rates. The $V_{d d}$ is no longer adequate. We, then construct a semi-empirical potential to describe the RET process. 3) At high densities, we show that the atomic orbital of $\left|n_{a}\right\rangle$ overlaps with that of neighboring atoms, and the electron-electron potential becomes prominent, resulting in much higher rates.
\end{abstract}

\section{Keywords}

Resonant Energy Transfer (RET), Molecular Auto-Ionization (MAI), Cold Rydberg Gas

\section{Introduction}

The relaxation of a cold Rydberg gas produced initially by exciting the atoms to 
Rydberg states $\left|n_{0}\right\rangle$, with the principal quantum number $n_{0} \gg 1$, has been the subject of much recent studies, both theoretically [1]-[7] and experimentally [8]. At low densities, the Rydberg atoms (RyA's) in the gas interact with each other by the dipole-dipole coupling $V_{d d}$. One of the prime breakdown mechanisms of the gas, mediated by $V_{d d}$, is the Penning-type molecular auto-ionization (MAI), in which the de-excitation energy of one RyA is transferred to the second one and ionizing it. However, at the density of $N_{A}^{e x p} \approx 10^{9} \mathrm{~cm}^{-3}$, the decay rates were observed [8] to be too large, by as much as a factor of two orders of magnitude or more of the MAI rates. Besides, a large number of low energy free electrons as well as readily field-ionizable excited state atoms were detected.

In this paper, we discuss the resonant energy transfer (RET), a Forster-type [9] [10] [11] process, in which a pair of RyA's shares its internal energies and creates new bound states, one higher and the other lower than the original state $\left|n_{0}\right\rangle$. This RET process is complementary to MAI and is always present if certain energy resonance conditions are satisfied. However, the RET process has been omitted in the past, because its rates are known to be very small compared to that of MAI, especially when $n_{0}$ is small and the density of the gas $N_{A}$ is low. By a simple model, we examine in detail the $n_{0}$ and density dependences of the RET rates as well as that of MAI. Especially for the RET, as the density increases, the dipole-dipole interaction $V_{d d}$ between the RyA's may no longer be valid and must be modified.

The density of a cold gas $N_{A}\left(\mathrm{~cm}^{-3}\right)$ defines the average separation $R_{N}$ between a pair of atoms, given by $R_{N}\left(a_{B}\right) \approx 1.9 \times 10^{8}\left[N_{A}\left(\mathrm{~cm}^{-3}\right)\right]^{-1 / 3}$. E.g. $N_{A}=10^{11} \mathrm{~cm}^{-3}$ gives $R_{N} \approx 4 \times 10^{4} a_{B}$, where $a_{B}$ is the Bohr radius which we set equal to one. Furthermore, the radius of the RyA is defined simply as $r_{0}=n_{0}^{2}$ and $r_{a}=n_{a}^{2}$, neglecting the factor $3 / 2$ and angular momentum dependence. In discussing the RET, the $r_{a T}=2 r_{a}$ is the important parameter [1] [3], and we simply define (a) the low density as $R_{N}>2 r_{a T}$, (b) the moderate density as $2 r_{a T}>R_{N}>r_{a T}$, and (c) the high density as $R_{N}<r_{a T}$.

\section{The MAI at Low Density}

For a pair of RyA's A and B, each in state $\left|n_{0}, l_{0}\right\rangle$, and neglecting the $l_{0}$ part, the two-body MAI involves the transition $\{i \rightarrow f\}$, where $[i]=\left[\left(n_{0}\right)_{A},\left(n_{0}\right)_{B}\right]$ and $[f]=\left[\left(n_{b}\right)_{A},(c)_{B}\right]$, and where $n_{b} \ll n_{0}$ and $(c)_{B}$ denote the ionized electron from $B$. At low densities and low temperature of interest here, the motion of the pair and van der Waal's attraction may be neglected. States are specified by [., .], while transitions are specified by $\{[..] \rightarrow[.]$.$\} .$

1) The MAI amplitude. The system Hamiltonian is defined as

$$
H=\left(K_{I, I I}+K_{1, I}+K_{2, I I}+V_{1, I}+V_{2, I I}\right)+\left(V_{1, I I}+V_{2,1}+V_{12}+V_{I, I I}\right) \equiv H_{0}+V
$$

where $I, I I$ denote the ions and 1, 2 denote the ionized electrons. While being attracted to each other by the van der Waal's potential (which is quadratic in $V_{d d}$ and adiabatic), the MAI proceeds as

$$
\{i \rightarrow f\}=\left\{\left(n_{0} \rightarrow n_{b}<n_{0}\right)_{A}\left(n_{0} \rightarrow c_{b}=\text { continuum }\right)_{B}\right\} \text { (MAI) }
$$


where simply $[i]=\left[n_{0}, n_{0}\right]$ and $[f]=\left[n_{b}, c_{b}\right]$; the transition (2.2) produces a free electron, an ion $I I=B^{+}$, and a bound atom A in state $\left|n_{b}\right\rangle$ with $n_{b} \ll n_{0}$. The energy conservation for (2.2) is

$$
E_{i}\left(=2 e_{0}\right)=E_{f}\left(=e_{n b}+e_{c b}\right)
$$

where $e_{0}=-1 / n_{0}^{2}$ Ry, $e_{n b}=-1 / n_{b}^{2}$ Ry, etc, and $e_{c b} \geq 0$ for ionization to take place. The highest allowed value $n_{b}^{x}$ of $n_{b}$ is obtained by setting $e_{c b}=0$ in (2.3), as

$$
n_{b}^{x}=n_{0} / \sqrt{2}, \text { (or the closest integer below it) }
$$

For $n_{0}=50$, we have $n_{b} \leq 35 \equiv n_{b}^{x}$.

The MAI amplitude (superscript ma) is defined, in the "prior" form, as

$$
M_{f i}^{m a}=\left(\Psi_{f}, V_{i} \Phi_{i}\right)
$$

where $(H-E) \Psi_{f}=0$, and $\left(H_{i}-E\right) \Phi_{i}=0$ with $H_{i}=H_{0}$ and $V_{i}=V$. In the Born approximation and at low densities, we let $\Psi_{f} \rightarrow \Phi_{f}$ with

$\left(H_{0}-E\right) \Phi_{f}=0$, while $V_{i} \rightarrow V_{d d}$, as

$$
M_{f i}^{m a} \approx\left(\Phi_{f}, V_{d d} \Phi_{i}\right)
$$

where

$$
V_{d d}=2\left[\left(\boldsymbol{r}_{1} \cdot \boldsymbol{r}_{2}\right)-3\left(\boldsymbol{r}_{1} \cdot \boldsymbol{\varepsilon}\right)\left(\boldsymbol{r}_{2} \cdot \boldsymbol{\varepsilon}\right)\right] / R^{3}, \boldsymbol{\varepsilon}=\boldsymbol{R} / R
$$

which makes the Born amplitude integral separable in $\boldsymbol{r}_{1}$ and $\boldsymbol{r}_{2}$.

2) The total MAI probabilities. The MAI transition probability per unit time is given by

$$
P_{f i}^{m a}=2 \pi\left|M_{f i}^{m a}\right|^{2}
$$

The $R^{-3}$ dependence of $V_{d d}$, causes the $P_{f i}^{m a}$ to decrease as $R^{-6}$.

The total MAI probability is obtained by summing the $P_{f i}^{d a}$ over the restricted set of final states [2], as

$$
\Gamma^{m a}=\sum_{n b=1}^{n_{b x}} P_{f i}^{m a}, f=\left(n_{b}, c_{b}\right)
$$

In (2.9), the sum $\sum_{n b}$ is over the range $1 \leq n_{b} \leq n_{b}^{x}$. (For type-setting, we used $\left.n_{b}^{x} \equiv n_{b x}, \quad c_{b} \equiv c_{n b}, \quad n b \equiv n_{b}\right)$. It has been established that the transition involving $n_{b}=n_{b}^{x}$ is the most dominant [1], as the both dipole matrix elements associated with $V_{d d}$ decrease as $n_{b}$ decreases from $n_{b}^{x}$ : the threshold dominance. Figure 1 illustrates two cases, $n_{b}=n_{b}^{x}$ and $n_{b}=n_{b}^{x}-1$.

It is important to point out that the states with $n_{b}^{\prime}>n_{b}^{x}$ are omitted in the sum (Equation (2.9)). They represent the RET, and thus the $n_{b}^{x}$ is the dividing point of the spectrum between the MAI and RET.

3) A Model Calculation. For the actual calculation of the $P_{f i}^{m a}$, we define the model parameters, as $n_{b}=10, n_{0}=20$, and $n_{a}=50=1 / k$ for the continuum function. (These parameters are not quite the actual MAI in specific cases, but represent the physics reasonably well, both for MAI and RET.) We let the orbital functions $\varphi=(u / r) \cdot Y_{l m}$. Since the $V_{d d}$ is separable and proportional to $R^{-3}$, the amplitude $P_{f i}^{m a}$ can be written in the form 


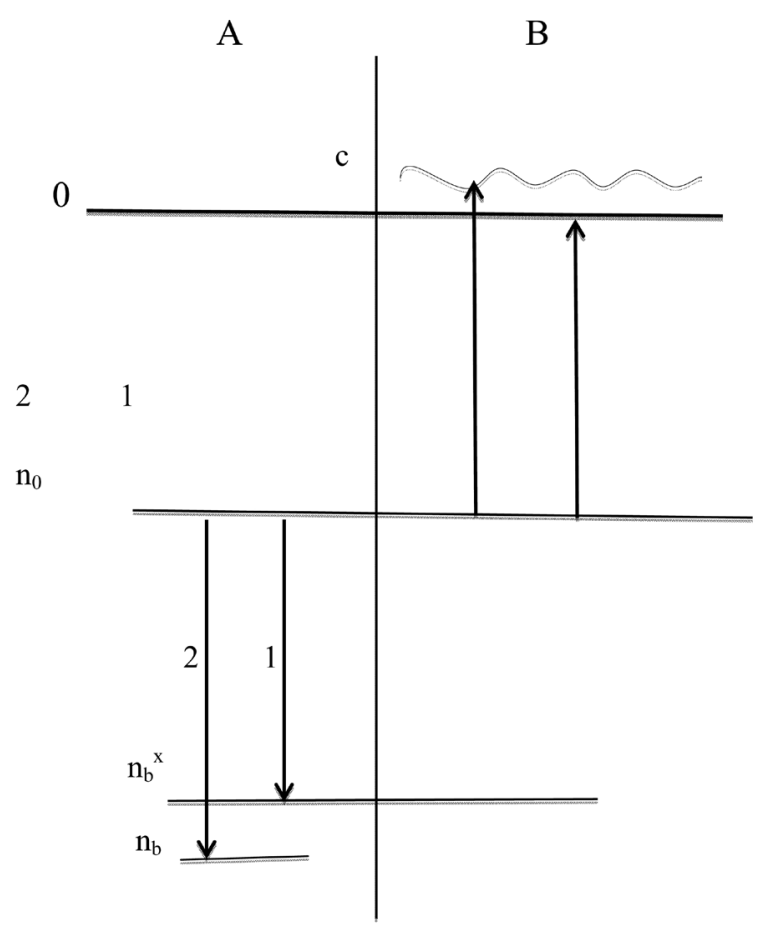

Figure 1. Illustration of levels and transitions for the MAI for two case: Case1: $n_{b}=n_{b}^{x}$, $e_{c b}=0 ;$ Case $2: n_{b}=n_{b}^{x}-1, e_{c b}>0$.

$$
P^{m a}\left(n l, n l \rightarrow n_{b} l^{\prime}, c_{b} l^{\prime}\right)=C_{p} I_{J} \cdot J / R_{N}^{6}
$$

where $C_{p}=8 \pi A_{y}^{2}$, with $A_{y}=\left|\int \mathrm{d} \Omega Y_{l m} Y_{l m+m^{\prime}} Y_{l^{\prime} m^{\prime}}^{*}\right|^{2}$ for $l^{\prime}=l \pm 1$. We have $A_{\mathrm{y}}=$ $1 /(4 \pi)$ for $l=0$ and $A_{\mathrm{y}}=1 /(5 \pi)$ for $I=1, m=0$. Explicitly, $C_{p 0}=1 /(2 \pi) \approx 0.16$ for $l=0$, e.g. and $C_{p 1}=8 /(25 \pi) \approx 0.11$ for $l=1$. We obtain

$$
\begin{gathered}
I_{J}\left(n l, n_{b} l^{\prime}\right)=\int r_{1} u_{n l} u_{n b l^{\prime}} \mathrm{d} r_{1}=3.19 \\
J\left(n l, c_{b} l^{\prime}\right)=\int r_{2} u_{n l} u_{c b l^{\prime}} \mathrm{d} r_{2}=7.47 \times 10^{4}
\end{gathered}
$$

Thus, with $R_{N}=r_{a T}=2 r_{a}=5.0 \times 10^{3}$ and $R_{N}^{6}=1.6 \times 10^{22}$,

$$
P^{m a} \approx C_{p 0} \times 1.5 \times 10^{-17}
$$

\section{The RET at Low Density}

In RET, a pair of RyA's, A and B, can exchange some of their internal energy, such that both atoms remain in the bound states of different n's; the process must satisfy the strict resonance energy condition. This can be satisfied either by an accidental matching of relevant energies, by a Stark shift, or by imposing an external electric field. An estimate of the Stark shift for example shows that, at a given density, high enough $n_{a}$ can satisfy the resonance condition. In the following, we simply assume that such conditions are met, and focus on the rates.

1) The RET probabilities (superscript re). The RET transition involves $[i]=\left[\left(n_{0}\right)_{A}\left(n_{0}\right)_{B}\right] \equiv\left[n_{0}, n_{0}\right]$ and $[j]=\left[\left(n_{b}^{\prime}\right)_{A},\left(n_{a}\right)_{B}\right]$, with $n_{b}^{\prime} \ll n_{0}$ and $n_{a} \gg n_{0}$. Thus, we have 


$$
\{i \rightarrow j\}=\left\{\left(n_{0} \rightarrow n_{b}^{\prime}=n_{b}^{x}+m^{\prime}\right)_{A}\left(n_{0} \rightarrow n_{a} \gg n_{0}\right)_{B}\right\}
$$

with $m^{\prime}=1,2, \cdots, n_{0}-n_{b}^{x}$, and the resonance energy conditions

$$
E_{i}=2 e_{n 0}=e_{n b^{\prime}}+e_{n a}=E_{j}, n_{b}^{\prime}>n_{b}^{x}
$$

The RET probability $P_{j i}^{r e}$ for $\{i \rightarrow j\}$ is given in the Born approximation as

$$
P_{j i}^{r e}=2 \pi\left|M_{j i}^{r e}\right|^{2} \text {, with } M_{j i}^{r e} \approx\left(\Phi_{j}, V_{d d} \Phi_{i}\right)
$$

A complete list of allowed $n_{a}$ for $n_{0}=50$ and $n_{b}^{x}=35$ is obtained from (Equation (3.2)) for the final state of RET $[j]=\left(n_{b}^{\prime}, n_{a}\right):(36,188),(37,120),(38,96)$, $(39,84), \cdots,(48,52),(49,51)$, provided (Equation $(3.2))$ is satisfied. For the maximum $n_{a}=188$, we have $r_{a} \approx n_{a}^{2} a_{B} \approx 16 r_{0}=3.5 \times 10^{4} a_{B}$; in this case, $R_{N}$ must be larger than $8 R_{0}$ to have the $V_{d d}$ applicable. Incidentally, we note that the RET for near-by states transitions, such as $(48,52)$ and $(49,51)$, have often been considered [11], but not for maximum $n_{a}$. (The RET rates for small $n_{0}$ and large $n_{a}$ are usually very small, but not for large $n_{0}<n_{\mathrm{a}}$.)

Generally, the maximum $n_{a}$ depends sensitively on the lowest $n_{b}^{\prime}$, and varies widely, roughly between 150 to 300 and more. For the general discussion, we simply take the typical value $n_{a}=200$. Since at $R_{N} \approx r_{a T}+r_{b^{\prime} T} \approx r_{a T}$, the "tails" of two orbital functions for $\left|n_{a}\right\rangle$ and $\left|n_{b}^{\prime}\right\rangle$ start to overlap each other [1] [3], and where the transition probabilities start to increase exponentially, the $r_{a T}$ is the very parameter that can be used to define the regions of different densities, as given in Section 1.

2) The total RET probability. The total probability that includes the on-shell part of the sum in $j=\left(n_{b}^{\prime}, n_{a}\right)$ is given by

$$
\Gamma^{r e}=\sum_{n b^{\prime}=n b x+1}^{n 0-1} P_{j i}^{r e}, j=\left(n_{b}^{\prime}, n_{a}\right),
$$

where the sum over $n_{b}^{\prime}$ is $n_{b}^{x}+1 \leq n_{b}^{\prime} \leq n_{0}-1$, provided the resonance condition is satisfied. (For typesetting we used $n b^{\prime}=n_{b}^{\prime}, n b x=n_{b}^{x}$, and $n 0-1=n_{0}-1$.) This is to be compared with the total MAI probability given by (2.4), $\Gamma^{d a}=\sum_{n b=1}^{n b x} P_{f i}^{d a}$, where $f^{\prime}=\left(n_{b}, c_{b}\right)$. Evidently, $\Gamma^{r e}$ complements $\Gamma^{d a}$ as the combined set $\left[n_{b}\right]_{f}+\left[n_{b}^{\prime}\right]_{j}$ is complete; the sum includes all values up to $n_{0}$ and the contributions from values above $n_{0}$ are already included as the states for $\mathrm{A}$ are exchanged to that of $\mathrm{B}$.

For the special case with hyper RyA, we can estimate the RET probability $P_{j i}^{r e}$ for a two-body system by comparing with the $P_{f i}^{m a}$ of MAI and adopting the quantum defect theory [12] [13]. To have a smooth analytic extrapolation over the threshold $e_{c}=0$, the continuum function in the $P_{f i}^{m a}$ is assumed energy-normalized [14] [15] [16]. For densities $R_{N}>2 r_{a}$, define

$$
Q \equiv P_{j i}^{r e}\left(n_{a}, n_{0}, R_{N}\right) / P_{f i}^{m a}\left(k_{n b}=\frac{1}{n_{a}}, n_{0}, R_{N}\right) .
$$

As expected, the quantum defect theory gives $Q=n_{a}^{-3}$, for $n_{0}<10$, but slowly increases with $n_{0}$, and becomes large. 
3) Our model calculation. Consider again the model with the parameters $n_{b^{\prime}}=$ $10, n_{0}=20$, and $n_{a}=50$. This corresponds roughly to one-half of the third set $[j]=\left(n_{b}^{\prime}, n_{a}\right)=(38,96)$. (Here, or simplicity, we take the same $n_{b^{\prime}}$ as $n_{b}$ of MAI.) We write $P_{f i}^{r e}$ as

$$
P_{j i}^{r e}\left(n_{0} l, n_{0} l \rightarrow n_{b}^{\prime} l^{\prime}, n_{a} l^{\prime}\right)=C_{p} I_{I} \cdot I / R_{N}^{6},
$$

where $C_{p}$ is defined earlier, and $l^{\prime}=l \pm 1$. Taking $l=0$, we obtain

$$
\begin{gathered}
I_{I}\left(n_{0} l, n_{b}^{\prime} l^{\prime}\right)=\int r_{1} u_{n_{0}} u_{n b^{\prime} l^{\prime}} \mathrm{d} r_{1}=3.19 . \\
I\left(n_{0} l, n_{a} l^{\prime}\right)=\int r_{2} u_{n_{0}} u_{n_{a} l^{\prime}} \mathrm{d} r_{2}=1.67 .
\end{gathered}
$$

Thus, with $R_{N}=r_{a t}=5.0 \times 10^{3}$ we have

$$
P_{j i}^{r e} \approx C_{p 0} \times 3.3 \times 10^{-22} \text {. }
$$

This is to be compared with Equation (2.12)

$$
P_{f i}^{m a}\left(n_{0} l, n_{0} l \rightarrow n_{b} l^{\prime}, k=n_{a}^{-1} l^{\prime}\right)=C_{p 0} \times 1.5 \times 10^{-17} .
$$

In general, $I_{I}>I_{J}$ and are nearly of same magnitudes for $n_{b}=n_{b}^{x}$ and $n_{b}^{\prime}=n_{b}^{x}+1$.

The main difference between the RET and MAI comes from $I$ and $J$. They are conveniently compared by

$$
Q=P^{r e} / P^{m a}=\left(I_{I} \cdot I\right) /\left(I_{J} \cdot J\right) \approx I / J,
$$

especially when $n_{b} \leq n_{b}^{x}$ and $n_{b}^{\prime} \geq n_{b}^{x}+1$ are close to each other and $n_{b}^{x} \gg 1$.

Our model calculation, with $V_{d d}$ and the parameters $n_{0}=20, n_{b}=10$, and $n_{a}=$ 50 , shows that $Q$ is very small for $n_{0}<n_{a} / 2$. Evidently, the RET is negligible for low $n_{0}$, and this may be the principal reason for neglecting it. More generally, because of the unusual dependence of $I\left(n_{0}, n_{a}\right)$ on $n_{0}$ for a fixed $n_{a}$ (Figure 2), $Q$ behaves approximately as

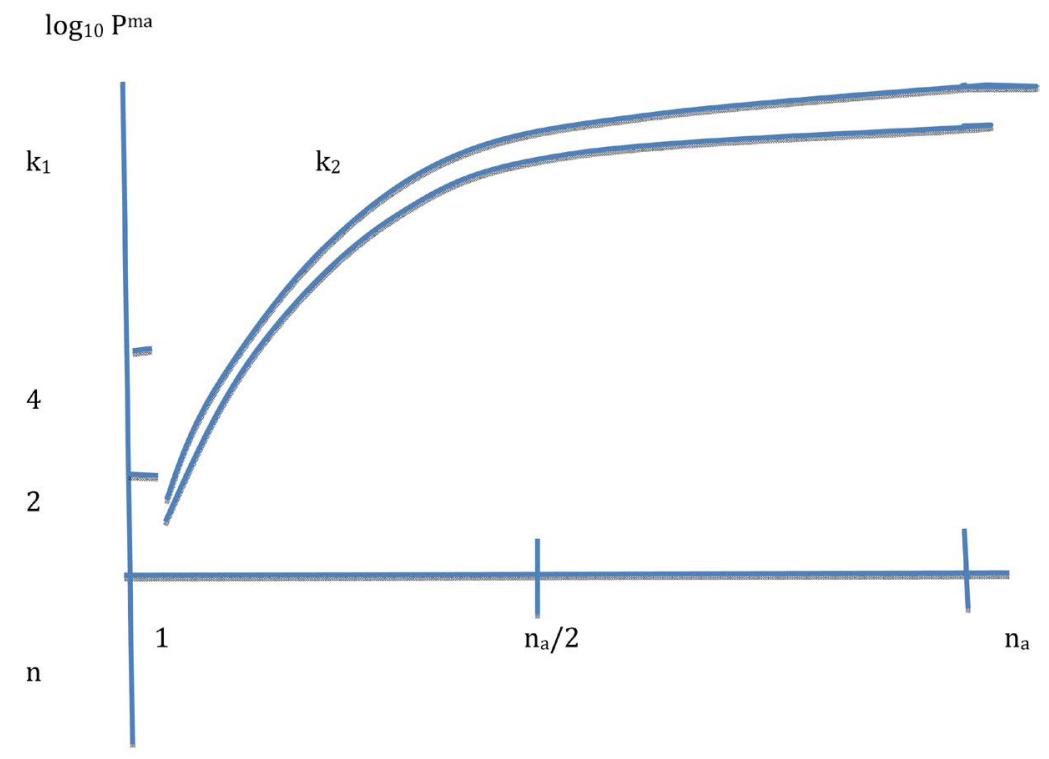

Figure 2. $P^{m a}: n_{0}$ and $k$ dependences where $k=1 / n_{a}$. 


$$
\begin{gathered}
n_{a}^{-3} \leq Q<n_{a}^{-2}, \text { for } 1 \leq n_{0}<n_{a} / 4 \quad(\mathrm{QDT}), \\
n_{a}^{-2}<Q<1, \text { for } n_{a} / 4<n_{0}<3 n_{a} / 4, \\
1<Q<n_{a}^{2}, \text { for } 3 n_{a} / 4<n_{0}<n_{a} .
\end{gathered}
$$

For applications, (3.10b) is the most relevant. The above trend continues to hold for larger $n_{a}$.

\section{The RET at High Densities}

The RET probabilities discussed in the previous section are for low density, with $R>2 r_{a T}$, where the dipole-dipole coupling $V_{d d}$ is effective. However, as the density increases and $R$ approaches $r_{a T}$, the $V_{d d}$ is no longer adequate in so far as treating the RET is concerned. By contrast, for the MAI, the $V_{d d}$ should be valid for $R_{N} \geq r_{a T} / 4 \geq R_{0}=4 r_{0}$. Thus, the result of Section 2 is unchanged; in fact, the MAI does not involve hyper-Rydberg states.

1) The RET amplitude (superscript h for high density) via $V_{12}$. We write the RET amplitude in the "post" form as

$$
M_{j i}^{r e-h}=\left(\Phi_{j}^{M}, V_{j} \Psi_{i}\right),
$$

where $\Phi_{j}^{M}$ now satisfies $\left(H_{j}-E\right) \Phi_{j}^{M}=0$, with $H_{j}=H-V_{12}$ and thus $V_{j}=V_{e e}=V_{12}$. Note that the $\Phi_{j}^{M}$ is a two-center molecular orbitals. In the Born approximation for the $\Psi_{i} \rightarrow \Phi_{i}$, we have (superscript $h$ for high density) for the post form

$$
M_{j i}^{r e-h}=\left(\Phi_{j}^{M}, V_{12} \Phi_{i}\right)
$$

and the RET probability is

$$
P_{j i}^{r e-h}=2 \pi\left|M_{j i}^{r e M}\right|^{2} .
$$

2) Evaluation of the amplitude integrals-approximations. The integrals involved in the $M_{j i}^{r e}$ are rather complex, and in the following, we make some simple approximations to obtain an estimate of the amplitude. Firstly, the molecular $\Phi_{j}^{M}$ is replaced by its LCAO (Linear Combination of Atomic Orbitals) form, $\Phi_{j}$ which is a solution of $H_{j}$; this will give only a crude estimate of the amplitude. Secondly, the variables involved in the $V_{12}=1 / r_{12}$ are complicated, as $r_{12}=\left|\boldsymbol{r}_{2 I}-\boldsymbol{r}_{1}\right|$ with $\boldsymbol{r}_{2 I}=\left|\boldsymbol{R}+\boldsymbol{r}_{2}\right| \equiv s_{2}$, or $r_{12}=\left|\boldsymbol{r}_{2}-\boldsymbol{r}_{1, I I}\right|$ with $\boldsymbol{r}_{1, I I}=\left|\boldsymbol{r}_{1}-\boldsymbol{R}\right| \equiv s_{1}$. We first set the dipole case, as $V_{12} \rightarrow Y_{1 m}(1) Y_{1 m}^{*}(2) r_{<}^{\prime} / r_{>}^{\prime 2}$, where $r^{\prime}=r_{2}$ or $s_{1}$. Second, we let all the orbital functions be in the form $\varphi=(u / r) \cdot Y_{l m}$. To further simplify the task, e.g., let $s_{l}$ to be strictly a function of $r_{l}$ and $R$, but not its angular dependence. Then, the angular parts become trivial, and we have

$$
P_{j i}^{r e-h}=C_{p} \times(1 / 4) \times\left|T^{r e}\right|^{2},
$$

where the $C_{p}$ was defined earlier, and the amplitude integral is given by

$$
T^{r e}=\int_{0}^{\infty} \mathrm{d} r_{2} u_{n l} u_{n_{a} l^{\prime}}\left\{\left(1 / r_{2}^{2}\right) \int_{0}^{r_{2}} \mathrm{~d} r_{1} s_{1} u_{n l} u_{n b^{\prime} l^{\prime}}+r_{2} \int_{r_{2}}^{\infty} \mathrm{d} r_{2} u_{n l} u_{n b^{\prime} l^{\prime}} / s_{1}^{2}\right\} \equiv T_{1}+T_{2}
$$

Various simplifying approximations are considered: (si) $s_{1}=r_{1}$, (sii) $s_{1}=2 n_{0}^{2}$, 
(siii) $s_{1}=2 n_{a}^{2}$. All three cases make the angular integrals simple.

We also define for convenience, $W_{1}=\int_{0}^{r_{2}} \mathrm{~d} r_{1} s_{1} u_{n l} u_{n_{a} l^{\prime}}$ and $W_{2}=\int_{r_{2}}^{\infty} \mathrm{d} r_{1} u_{n l} u_{n b^{\prime} l^{\prime}} / s_{1}^{2}$ Then, e.g. for (si) $W_{1} \sim \int_{0}^{r_{2}} \mathrm{~d} r_{1} r_{1} u_{n l} u_{n_{a} l^{\prime}}$ and $W_{2} \sim \int_{r_{2}}^{\infty} \mathrm{d} r_{1} u_{n l} u_{n b^{\prime} l^{\prime}} / r_{1}^{2}$, etc.

The results for the model are:

si: $T_{1}=7.11 \times 10^{-7}, T_{2}=-1.68 \times 10^{-7} ; T^{2}=2.9 \times 10^{-13}$, Molecular

sii: $T_{1}=2.54 \times 10^{-5}, T_{2}=5.73 \times 10^{-9} ; T^{2}=6.45 \times 10^{-10}, n_{0}$ Overlapping

siii: $T_{1}=1.59 \times 10^{-4}, T_{2}=1.47 \times 10^{-10} ; T^{2}=2.53 \times 10^{-8}, n_{a}$ touching.

\section{Summary and Conclusion}

We have presented the RET as the potentially important process that affects the relaxation of cold Rydberg gas. This process has been neglected in all the previous studies of the decay of the gas, presumably because of its extreme low rates at small $n_{0}$. Our present study has shown the importance of the RET, especially because of the large physical size of the hyper-Rydberg state; it basically changes the effective density by many folds.

The MAI is shown to be the dominant process at low density, $R_{N}>2 r_{a}$, where the $V_{d d}$ is effective, while the RET is always present, but at very low probabilities. The $n_{0}$ dependence of the $P^{r e}$ and $P^{d a}$ is studied in detail; for the initial excited state $\left|n_{0}\right\rangle$ not too high, $1 \leq n_{0}<3 n_{a} / 4$. The ratio $Q=P^{r e} / P^{d a}$ is found to be very small, of the order of $n_{a}^{-3}$ to $n_{a}^{-1}$. The lower limit of $Q$ follows from the quantum defect theory, which is approximately valid for $n_{0}<10$, but starts to break down for higher $n_{0}$. As the $n_{0}$ approaches $n_{a}, Q$ increases to one, and grows rapidly, to as much as $n_{a}^{2}$.

For the cold Rydberg gas at moderate density, $r_{a T}<R_{N}<2 r_{a T}$, the wave function $\varphi_{a}$ starts to overlap with the neighboring RyA's, and the $V_{d d}$ is no longer applicable. It is replaced by the electron-electron interaction $V_{e e}$. The modified RET probabilities, $P_{j i}^{r e-M}$, are estimated in several approximations, all of which indicate the resulting $Q^{M}$ to be much larger than the $Q$, of the order to $n_{a}$. As expected, the $P_{j i}^{r e-h}$ at high density is much larger, with the overlap of orbital wave functions.

The RET can create a hyper RyA of large size, which in turn immediately forms a giant auto-ionizing clusters, enveloping many near-by atoms, and contains a huge amount of excess internal energies, making it highly unstable. Multiple production of clusters in the gas leads to a cascade decay of the gas. This problem requires a careful analysis with rate equations.

The dominance of states near the ionization threshold both in the MAI and RET processes yields multitude of low energy free electrons, via MAI, and many weakly bound electrons, via RET. These results are consistent with the experimental observation [8].

\section{Acknowledgements}

This research work was supported by the National Science Foundation (NSF). The award number is 1901397. 


\section{Conflicts of Interest}

The authors declare no conflicts of interest regarding the publication of this paper.

\section{References}

[1] Hahn, Y. (2000) Journal of Physics B, 33, L655. https://doi.org/10.1088/0953-4075/33/20/101

[2] Amthor, T., et al. (2009) The European Physical Journal D, 53, 329. https://doi.org/10.1140/epjd/e2009-00119-4

[3] Kiffner, M., et al. (2016) Journal of Physics B, 49, Article ID: 204004. https://doi.org/10.1088/0953-4075/49/20/204004

[4] Robicheaux, F. (2005) Journal of Physics B, 38, S333. https://doi.org/10.1088/0953-4075/38/2/024

[5] Amthor, T., et al. (2007) Physical Review A, 76, Article ID: 054702.

[6] Amthor, T., et al. (2007) Physical Review Letters, 98, Article ID: 023004.

[7] Robicheaux, F., et al. (2014) Physical Review A, 90, Article ID: 022712.

[8] Tanner, J.T., et al. (2008) Physical Review Letters, 100, Article ID: 043002.

[9] Forster, Th. (1948) Annalen der Physik, 437, 35. https://doi.org/10.1002/andp.19484370105

[10] Maineult, W., et al. (2016) Journal of Physics B, 49, Article ID: 214001. https://doi.org/10.1088/0953-4075/49/21/214001

[11] Comparat, D. and Pillet, P. (2010) Journal of the Optical Society of America B, 27, A208. https://doi.org/10.1364/JOSAB.27.00A208

[12] Hahn, Y. (2002) Physics Letters A, 293, 266. https://doi.org/10.1016/S0375-9601(01)00854-4

[13] Bethe, H.A. and Salpeter, E.E. (1957) Quantum Mechanics of One- and Two-Electron Atoms. Springer-Verlag, Berlin, 264. https://doi.org/10.1007/978-3-662-12869-5

[14] Hahn, Y. (1977) Physical Review Letters, 39, 82. https://doi.org/10.1103/PhysRevLett.39.82

[15] Hahn, Y. (1985) Advances in Atomic and Molecular Physics, 21, 123. https://doi.org/10.1016/S0065-2199(08)60142-6

[16] Seaton, M.J. (1955) Comptes Rendu, 240, 1317. 